\title{
Hyperspectral Acquisition Technology Based on Compressed Sampling in Spatial Domain
}

\author{
Shuyao Tian ${ }^{1,3}$, Zhen Zhao ${ }^{1 *}$, Tao Hou ${ }^{2}$, Liancheng Zhang ${ }^{1}$ \\ ${ }^{1}$ College of Electronics and Control Engineering, North China Institute of Aerospace Engineering, \\ Langfang, China \\ ${ }^{2}$ Beijing Aerospace Xinli Technology Co., Ltd, Beijing, China \\ ${ }^{3}$ Hebei Electric Vehicle Charging Technology Innovation Center, China
}

Received: July 17, 2021. Revised: December 3, 2021. Accepted: January 10, 2022. Published: January 12, 2022.

\begin{abstract}
In the hyperspectral imaging device, the sensor detects the reflection or radiation intensity of the target at hundreds of different wavelengths, thus forming a spectral image composed of hundreds of continuous bands. The traditional processing method of sampling first and then compressing not only cannot fundamentally solve the problem of huge amount of data, but also causes waste of resources. To solve this problem, a spectral image reconstruction method based on compressed sampling in spatial domain and transform coding in spectral domain is designed by using the sparsity of single-band two-dimensional image and the spectral redundancy of spatial coded data. Based on Bayesian theory, a compressed sensing measurement matrix of adaptive projection is proposed. Combining these two algorithms, an adaptive Grouplet-FBCS algorithm is constructed to reconstruct the image using smooth projection Landweber. Experimental results show that, compared with existing image block compression sensing algorithms, this algorithm can significantly improve the quality of image signal reconstruction.
\end{abstract}

Keywords - Spatial domain compression; Hyperspectrum; Compressed sensing; Texture features; adaptive algorithm

\section{INTRODUCTION}

Qpectral technology and imaging technology are two Oimportant fields in photoelectric detection technology, and their respective development is maturing and they are widely used. In 1960s, remote sensing technology rose. In the field of remote sensing, people want not only the spatial information of the detected target, but also the high-resolution spectral information of the target while detecting the high-resolution spatial information [1]. Driven by the application demand, the imaging optical instrument and spectral detection instrument are creatively combined in the remote sensing field, and the spectral imaging technology is formed [2-3].

Although hyperspectral imaging has great potential for civil and military use, because hyperspectral image is a kind of 3D image data, its data capacity is very large. Especially, when the inter-spectral resolution of hyperspectral images is improved, the amount of image data will increase dramatically [4]. The amount of data in hyperspectral images is very large. In order to ensure the efficient transmission and storage of massive hyperspectral images, its high-quality image compression sampling coding has become one of the important key technologies [5-6]. Literature [7] thinks that the traditional data compression is relatively low and the sampling amount is large, so the compressed sensing algorithm is introduced to reduce the sampling rate of large data, and at the same time reduce the data amount and improve the calculation efficiency. However, the performance of sparse models used in compressed sensing algorithms is less considered. [8] According to the existing characteristics of hyperspectral images, the coding difficulty is simplified by regularization projection, and the reconstruction quality of signal-to-noise ratio optimization is improved, but the model used for coding is not considered. When studying hyperspectral image reconstruction in Literature [9-10], the compressed sensing technology was applied to edge information reconstruction, and a joint prediction algorithm was proposed for compressed reconstruction in spatial spectrum domain, which reduced the computational complexity and increased the computational speed by projection of convex sets alternately. This method achieved good reconstruction results in compressed reconstruction of hyperspectral images. [11] Considering that compressed sensing algorithm needs high-quality sparse model, in order to improve the quality of hyperspectral image reconstruction and the ability of the algorithm to accurately recover information, the structure information is estimated a priori, which significantly improves the accuracy of image reconstruction by the proposed algorithm. At the same time, it has been well verified when applied to images with noise background [12-14], a lossy compression method based on in-band prediction and inter-band fractal coding is proposed. Literature [15] proposed clustering differential pulse coding for lossless compression of hyperspectral images in spectral domain.

In order to realize spectral imaging and decoding reconstruction, this paper proposes a spectral image reconstruction method based on spatial domain compression sampling. In this paper, the spatial dimension information of hyperspectral data is fully mined and utilized, so that the 
classification results of hyperspectral data are optimized to a certain extent. Compared with the typical hyperspectral classification methods combined with spatial domain information, the advantages of this method are that it relies less on prior knowledge in the optimization process, and it can achieve better performance when classifying targets in complex background.

\section{ORIGINALITY}

For the image compression sensing algorithm, the traditional compression sensing processing only uses the sparsity of the image signal as a priori, and does not consider the distribution of the image sparsity coefficient, so the sampling rate cannot be reasonably allocated by using the characteristics of the signal itself. All areas in the same image signal are collected at the same sampling rate, which leads to great differences in the reconstruction effect of image blocks with different sparsity in the same projection domain, and the details are not prominent.

In this paper, based on compressed sampling in spatial domain, the Grouplet transform is more suitable for actual images in scientific research through adaptive threshold denoising, instead of idealized threshold setting. Self-adaptively preprocess the noise in each band of hyperspectral image, so that the band affected by noise has better image quality and clearer image surface after compression and reconstruction.

Adaptive sampling compressive sensing algorithm based on spatial and directional features. If there is a priori, the spatial frequency of the image block is used to determine the type of the image block, and the basic sampling rate is obtained, and then the sampling rate is adjusted by the statistical characteristics of the direction coefficient in the transform domain.

Aiming at the problem that the traditional compressed sensing algorithm can not adaptively adjust the sampling rate according to the signal characteristics in image processing applications, this paper proposes an adaptive sampling compressed sensing algorithm with prior information. This paper mainly studies the problem of adaptive sampling rate allocation using statistical features of image transform domain coefficients and texture features in spatial domain.

\section{RESEARCH METHOD}

Hyperspectral imaging technology is based on many narrow-band image data technologies, which combines imaging technology with spectral technology, detects the two-dimensional geometric space and one-dimensional spectral information of the target, and obtains continuous and narrow-band image data with high spectral resolution.

\section{A. Sparse Transformation and Image Coding}

As we all know, the premise of signal compression sampling is that the signal is compressible. The compressibility of the signal contains two meanings: first, the signal itself is sparse, that is, most of the coefficients in the signal are zero, and only a few non-zero coefficients exist; Second, from a broader perspective, the signal itself may not be sparse, but its projection on a certain space is sparse.

Compressed sensing, also known as compressed sampling or compressed sensing, is a method of capturing and representing compressible signals at a frequency far lower than Nyquist sampling frequency (Figure 1). The original signal is reconstructed by solving the 0 - norm optimization problem. Compressed sampling:

$$
y=\Phi x
$$

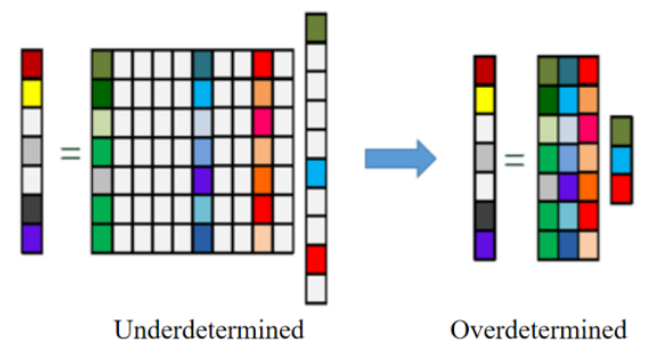

Figure 1. Sparse signal compression sampling

Any signal in $\mathfrak{R}^{N}$ space can be represented by the linear combination of $N$-dimensional orthogonal bases $\left\{\Psi_{i}\right\}_{i=1}^{N}$. With $\mathrm{N} \times \mathrm{N}$ basis matrix $\Psi=\left[\Psi_{1}\left|\Psi_{2}\right| \cdots \Psi_{N}\right]$, each column of the basis matrix is a basis in $\Psi_{i}$ set. $x$ can be expressed as

$$
x=\sum_{i=1}^{N} s_{i} \Psi_{i}
$$

$S$ is $\mathrm{N} \times 1$ dimensional column vector, and the weight coefficient is $s_{i}=\left\langle x, \Psi_{i}\right\rangle$.

If only $K$ coefficients in $N$-dimensional signal $S$ are not zero, and other coefficients are all zero, then the coefficients are $K$ sparse [16-17].

The premise of compressed signal is compressible. Assuming that the sparsity of the $N$-dimensional signal $S$ is $K$, under the framework of compressed sensing, the $M$ -dimensional observation vector $y$ is obtained by observing for $M$ times $(K<M<N)$, and the observed signal $x$ can be accurately reconstructed through the projection matrix $\Phi$ and the observation vector $y$.

The image is compressible, and sparse decomposition can be obtained by projecting it into the transform domain. A good image compression algorithm can't be separated from a good sparse transformation. Similarly, the compressed sampled signal can't be separated from a good sparse basis in the reconstruction process. Figure 2 shows sparse decomposition of signals. 


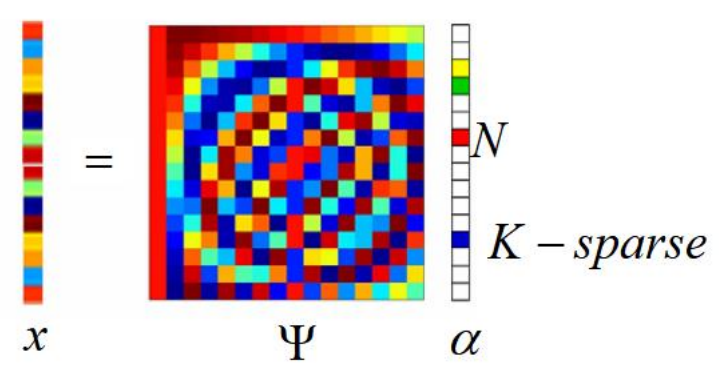

Figure 2. Sparse decomposition of signals

\section{B. Data Characteristics of Hyperspectral Imaging}

In the field of image research, to study image compression algorithms, it is necessary to study the statistical characteristics of images, that is, to study the correlation of images. As hyperspectral imaging is a typical three-dimensional data cube, the correlation of hyperspectral imaging is not only spatial correlation, but also strong inter-spectral correlation [18].

The autocorrelation of hyperspectral imaging image features, that is, the spatial correlation between different pixels in hyperspectral imaging in the same band, should be explained in the physical sense before studying. When the autocorrelation coefficient is 1, it can be seen that the autocorrelation function represents the spatial correlation of hyperspectral imaging in a single band.

In order to quantitatively analyze the spectral correlation of images, we introduce the cross-correlation function $h(l, k)$ to express it, and the formula is

$$
h(l, k)=\iint f(x, y) f(x+l, y+k) d x d y
$$

It is normalized and discretized, including:

$$
h(l, k)=\frac{\sum_{x=1}^{M} \sum_{y=1}^{N} f(x+l, y+k) g(x, y)}{\sqrt{\sum_{x=1}^{M} \sum_{y=1}^{N}[f(x, y)]^{2}[g(x, y)]^{2}}}
$$

In the formula, $f(x, y), g(x, y)$ respectively represents the gray level of pixels corresponding to $(x, y)$ in two adjacent hyperspectral imaging, and $l, k$ respectively represents the position change values of rows and columns of pixels. When $l=k=0, h(l, k)$ is called the cross-correlation coefficient between two images, which is denoted as $\varphi$.

In hyperspectral imaging, the correlation between hyperspectral imaging in two adjacent bands is very strong. This spectral correlation is unique to hyperspectral imaging data cube, so the statistical characteristics of this data should be fully utilized in hyperspectral imaging processing and compression [19]. Several bands of hyperspectral imaging Canal are selected for testing, and their spatial correlation and inter-spectral correlation are calculated. Figure 3 shows the spatial correlation coefficients of the 40th and 60th bands.

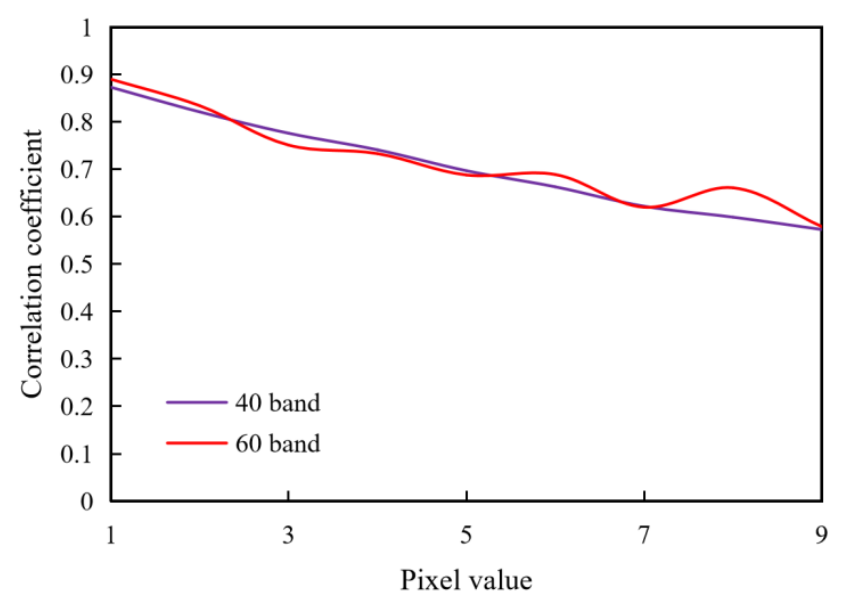

Figure 3. Spatial correlation coefficients of the 40th and 60th bands.

General interferometric hyperspectral imager, whether temporal, spatial or spatio-temporal modulation, finally obtains interferometric data cube as shown in Figure 4.

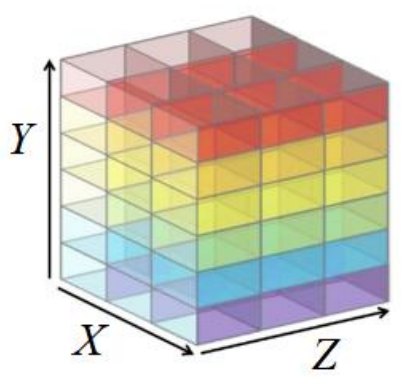

Figure 4. Typical interference data cube

It can be seen from Figure 4 that the interference data cube collected in real time is gray-white gray scale. Therefore, the statistical characteristics of interferometric hyperspectral imaging will be different from dispersive hyperspectral imaging and grating hyperspectral imaging. Among them, there is spatial correlation between $X, Y$ directions and spectral correlation between $Z$ directions.

In theory, interference hyperspectral imaging has good spatial correlation and inter-spectral correlation, but in practice, due to the sampling of optical path difference and spatial resolution, the dynamic range of inter-frame correlation of interference images is relatively large. If the collected images are large similar objects, the inter-frame correlation is very strong, but if not, the inter-frame correlation will not be strong. Similarly, the dynamic range of correlation in $X$ direction is also relatively large. The correlation among the columns reflects the correlation of spectral dimension, which is relatively stable for spectral dimension. 


\section{Compression Coding}

According to CS theory [20], each observation can be regarded as the inner product of the row vector of the measurement matrix and the vector to be measured, and each observation value contains part of the global information of the data to be measured. Schmidt orthogonalization method is used to preprocess the measurement matrix, which does not affect the randomness of the measurement matrix and weakens its correlation [21].

Given spectral image data $X=\left\{X_{i}\right\}_{i=1}^{L} \in R^{N \times N \times L}$, where $L$ is the number of bands, $X_{i}$ is the two-dimensional image of band $i$, and $N$ is the image size. The measurement matrices $A$ and $B$ are used to observe the rows and columns of the image independently [22], and the observation model is obtained

$$
Y_{i}=A X_{i} B^{T}
$$

In which: $A, B \in R^{M \times N}, M$ is the observed quantity; $Y_{i} \in R^{M \times M}$ is compressed sampling data. At this time, the measurement matrix needs $2 M N$ storage space. Define the spatial sampling rate as

$$
R_{s p a}=\frac{M^{2}}{N^{2}}
$$

Information is the key to the subsequent application of spectral image detection information. In order to ensure the accuracy of spectral reconstruction and improve the accuracy of subsequent applications, this study uses different random Gaussian matrices as the measurement matrix of each band, that is, three-dimensional coding strategy is adopted to enhance the randomness between spectra of sampling coding, thus improving the quality of spectral reconstruction. KL transform is used to remove inter-spectral redundancy after compression sampling in spatial domain. So that the energy of encoded data is concentrated in a few principal components, and its core is eigenvalue decomposition of covariance matrix of zero-mean data [23].

The two-dimensional matrix form of compressed sampled data $\left\{Y_{i}\right\}_{i=1}^{L} \in R^{N \times N \times L}$ in spectral spatial domain is $Y=\left[y_{1}, y_{2}, \cdots, y_{M^{2}}\right]^{T} \in R^{M^{2} \times L} \quad, \quad$ where $y_{j} \in R^{L}\left(1 \leq j \leq M^{2}\right)$ is the $j$ th measured value and $\bar{y}=\frac{1}{M^{2}} \sum_{j=1}^{M^{2}} y_{j}$ is the mean value of compressed sampled data in spatial domain, so the covariance matrix of sampled data in spatial domain is

$$
C=\frac{1}{M^{2}-1} \sum_{j=1}^{M^{2}}\left(y_{j}-\bar{y}\right)^{T}\left(y_{j}-\bar{y}\right)
$$

Eigenvalue decomposition is carried out on covariance matrix $C$ to obtain eigenvalue matrix $D$ and eigenvector matrix $V$, which satisfy the following requirements:

$$
D=V^{T} C V
$$

In which the diagonal elements of $D$ are eigenvalues.

After eigenvalue decomposition, the eigenvectors corresponding to $K$ significant eigenvalues are adaptively selected to form the transformation matrix $P \in R^{L \times K}$, which satisfies the following requirements

$$
c=\frac{\sum_{i=1}^{K} \lambda_{i}}{\sum_{i=1}^{L} \lambda_{i}} \geq 0.99,1 \leq K \leq L
$$

In which $\lambda_{i}$ is the eigenvalue. At this time, due to the randomness of the measurement matrix in spatial domain, the number of significant eigenvalues of KL transform in spectral domain is not fixed.

The specific form of KL transform in spectral domain is $\widetilde{Y}=Y P$, and the data to be transmitted at this time includes mean $\bar{y}, \mathrm{KL}$ transform matrix $P$ and reserved principal component $\widetilde{Y}$, so the compression ratio $R_{\text {spe }}$ of KL transform in spectral domain is

$$
R_{s p e}=\frac{M^{2} K+L K+L}{M^{2} L} \approx \frac{K}{L}
$$

\section{Mathematical model of hyperspectral compression} sampling.

Any hyperspectral image contains a lot of redundant information and some noises, but these redundant information values are usually random and often mixed with the pixel values of the image. Because the noise and redundancy in hyperspectral images will affect the sparse representation of images, thus affecting the subsequent compression reconstruction, and the accuracy of sparse representation is the prerequisite for the accuracy of compression reconstruction, the process of removing these redundant information and noise has become the first step in image processing.

This section will be combined with the Grouplet algorithm with adaptive threshold to form an adaptive Grouplet-FBCS (AGrouplet-AFBCS) algorithm [24-25]. And the adaptive threshold Grouplet algorithm is combined with the compressed sensing algorithm to form the adaptive threshold Grouplet-CS (AGrouplet-CS) algorithm.

Smooth projection Landweber iterative compressed sensing reconstruction algorithm based on block measurement is an iterative threshold algorithm for block compressed sensing measurement, which has good reconstruction performance and fast convergence speed. 
Some processing procedures can be added to optimize the algorithm in the iterative process, and has strong scalability. It is applied to the decoding end of distributed compressed video sensing to decode and reconstruct video. Because compressed sensing measurement based on block can effectively reduce the computational complexity of coding end and memory consumption of measurement matrix, and because of the small amount of data each time, the network transmission has good real-time performance. In the iterative process, optimization criteria can be added or the statistical correlation of adjacent frames can be used to assist algorithm reconstruction, which reduces the blocking effect and improves the visual effect of the picture.

(1)Adaptive threshold theory of orthogonal Grouplet transformation.

The Bayesian Shrink soft threshold algorithm is introduced into the thresholding process of Grouplet transform, and the Grouplet transform algorithm with adaptive threshold is constructed to denoise hyperspectral images in image preprocessing. The soft threshold function $\eta_{T}$ is the contraction function, and the formula is

$$
\eta_{T}=\operatorname{sgn}(x) \cdot \max (|x|-T, 0)
$$

The method of introducing generalized Gaussian prior function into Bayesian theory to calculate the optimal threshold has been introduced into scientific research projects by scholars at home and abroad. And the signal and Gaussian noise are transformed by generalized Gaussian distribution, and then calculated by soft threshold formula, the optimal threshold can be obtained

$$
\begin{gathered}
T\left(\sigma_{x}\right)=\frac{\hat{\sigma}_{l}^{2}}{\sigma_{x}} \\
\hat{\sigma}_{l}=\frac{\operatorname{Media}(Y)}{0.6745} \\
\sigma_{x}=\sqrt{\max \left(\hat{\sigma}_{y}^{2}-\hat{\sigma}_{l}^{2}\right)} \\
\hat{\sigma}_{y}^{2}=\frac{1}{M} \sum_{k=1}^{M} Y^{2}
\end{gathered}
$$

$\hat{\sigma}^{2}$ is the variance of noise, $\sigma_{k}$ is the estimated value of variance of noise, $k$ is the number of decomposition layers, $Y$ is the coefficient value of high-frequency diagonal, and $\hat{\sigma}_{y}^{2}$ represents the average value of high-frequency subband diagonal $Y^{2}$. The above two variance parameters are obtained from the measured data of each subband.

Generalized Gaussian distribution is the information coefficient carried by natural images described by shape parameter $\beta$.

$$
\begin{gathered}
\beta(x)=C\left(\sigma_{x}, \beta\right) \exp \left\{-\left[\alpha\left(\sigma_{x}, \beta\right)|x|\right]^{\beta}\right\} \\
\alpha\left(\sigma_{x}, \beta\right)=\sigma_{x}^{-1}\left[\frac{\Gamma(3 / \beta)}{\Gamma(1 / \beta)}\right]^{1 / 2} \\
C\left(\sigma_{x}, \beta\right)=\frac{\beta \cdot \alpha\left(\sigma_{x}, \beta\right)}{2 \Gamma(1 / \beta)} \\
\Gamma(t)=\int_{0}^{\infty} e^{-u} u^{t-1} d u
\end{gathered}
$$

(2)Adaptive sampling compressive sensing algorithm based on spatial and directional features

First, calculate the initial value $x^{(0)}$, and then iterate using formulas (20) and (21)

$$
\begin{gathered}
\varphi^{(t)}=x^{(t)}+\frac{1}{\gamma} M^{T}\left(y-M x^{(t)}\right) \\
x^{(t+1)}= \begin{cases}\varphi^{(t)} & \text { if }\left|\varphi^{(t)}\right|>\tau^{(i)} \\
0 & \text { otherwise }\end{cases}
\end{gathered}
$$

Where $\gamma_{\text {is }}$ the size factor, and its size is the maximum eigenvalue of $M^{T} M$. It can be clearly seen from formulas (20) and (21) that PL(Projected Landweber) algorithm belongs to IST algorithm in convex relaxation.

In order to remove the blocking effect, SPL (Projected Land Weber Reconstruction) algorithm combines PL algorithm with smooth filtering, that is, the image is subjected to Weiner filtering once before each iteration [25]. In each iteration, Bivariate Shrinkage algorithm is used for threshold processing [26].

For smooth image blocks, there is little difference in statistical characteristics of sub-band coefficients in different directions, so there is no need to adjust the sampling rate, that is, $S_{i, l}^{\prime}=\max \left(S_{i, l}^{\prime}, 0.10\right)$. Fig. 5 shows the sampling schematic diagram of smooth block and texture block, and $L=1$ is taken for convenience of observation.

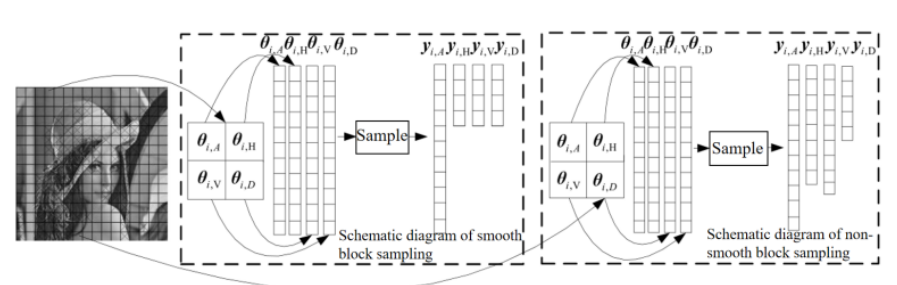

Figure 5. Schematic diagram of sampling when $L=1$

It is worth noting that the image block size in this section is only $32 \times 32$, and the number of wavelet transform layers $L=1$ or $L=2$ is more appropriate. When $L=1$ is used, the image reconstruction effect is better, but because the base band coefficient is completely reserved, the overall sampling rate must be greater than $T=1 / 4^{L}$, that is, 0.25 . 
When the target sampling rate is $S_{T}<0.25, L>1$, meanwhile, the texture block can't use the statistical features of wavelet coefficients to adjust $S_{i, j}^{\prime}$ well, so it can abandon the fine tuning step and use $S_{i, l}^{\prime}=\max \left(S_{i, l}^{\prime}, 0.10\right)$.

Therefore, when $S_{T}>T, v$ is set to 1 ; When $S_{T} \leq T$, $L=2$, sampling steps are shown in Figure 6 .

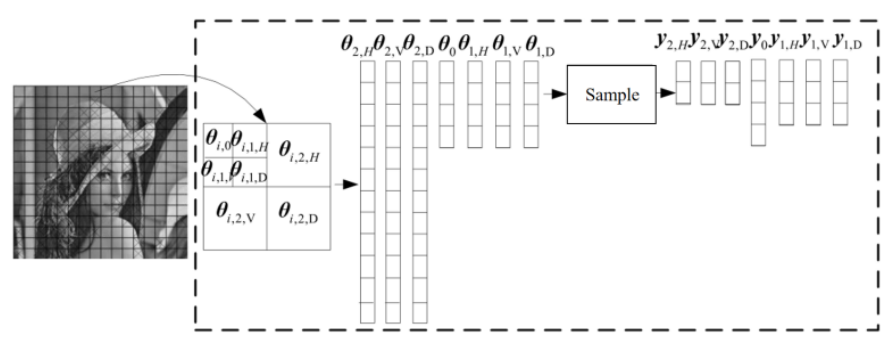

Figure 6. Schematic diagram of sampling when $S_{T} \leq T$

\section{(3)Algorithm flow}

The image is divided into blocks $x_{i}$ with the size of $B \times B$, and its spatial frequency is calculated respectively. The type of each block is judged, and the corresponding basic sampling rate $S_{i}$ is obtained.

And if $S_{T} \leq T$, performing two-layer wavelet decomposition on the image block, $\theta_{i}=\Phi x_{i}$, and obtaining a first-layer measured value $y_{i, l}=M_{i, l} \theta_{i, l}$, wherein the sampling rate is $S_{i, l}^{\prime}=\max \left(S_{i, l}^{\prime}, 0.10\right)$.

If $S_{T}>T$, the image block is decomposed by single-layer wavelet, $\theta_{i}=\Phi x_{i}$. And if $x_{i}$ belongs to a smooth block, obtaining a measured value $y_{i, l}=M_{i, s} \theta_{i, l}$, wherein $M_{i, s}$ is $m_{i, l} \times B^{2}, m_{i, l}=S^{\prime} B^{2}$; And if $x_{i}$ belongs to the texture block, obtaining the observed value $y_{i, j, l}=M_{i, j, l} \theta$.

Finally, according to formula (20) and formula (21), the SPL algorithm is used to reconstruct the image.

\section{RESULT ANALYSIS}

In the experiment, the hyperspectral images Indian Pines and Ribeira were selected, and all bands with a spatial size of $180 \times 180$ were taken as experimental data. The hyperspectral image Crown was selected for comparison with previous chapters, so pixels with a size of $64 \times 64$ in all bands of the image were intercepted. In the process of sparse representation of hyperspectral images by Grouplet-CS transform basis with adaptive threshold, pre-denoising is carried out in advance through adaptively set threshold, and whether the images are affected by noise has obvious differences on the reconstruction results.
A. Compression reconstruction errors of different compression ratios.

Ribeira data is noisy image data, which undergoes sparse reconstruction by adaptive Grouplet transform. The reconstruction error fluctuates obviously at different compression ratios, and the reconstruction error in the same band decreases significantly with the increase of compression ratio, as shown in Figure 7. At the same time, in the reconstruction process of compressed sensing, the image signal and noise are not estimated a posteriori, and the sparse model is not projected adaptively. Therefore, the intensive reading of the reconstructed image will be affected by noise, and the reconstruction accuracy of images in different bands is quite different.

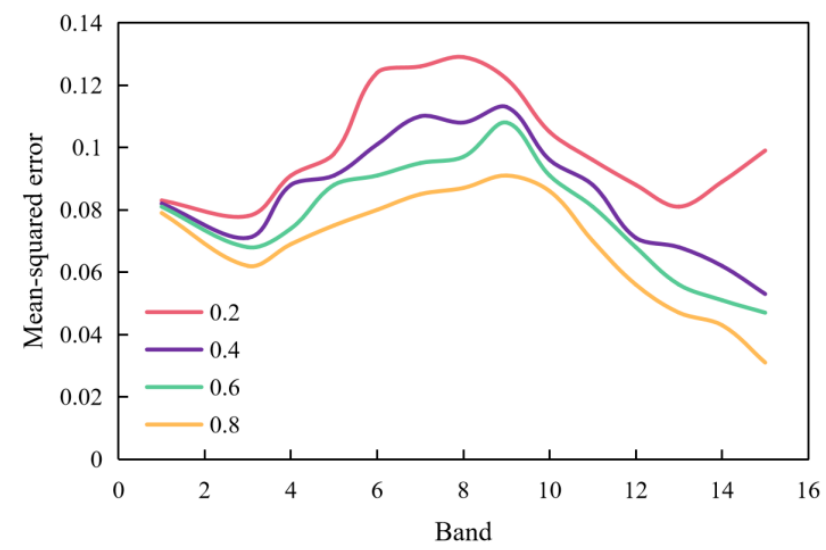

Figure 7. Compression reconstruction errors of Ribeira data with different compression ratios

According to Fig. 8, the overall reconstruction accuracy of two $3 \mathrm{~d}$ data is quite different, but both of them decrease with the increase of compression ratio. The error of Ribeira data mainly affected by noise is obviously higher than that of Indian Pines data with zero noise.

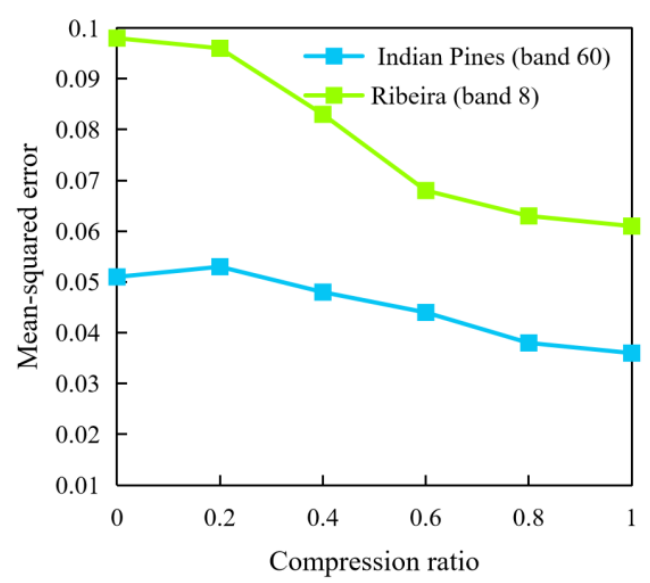

Figure 8. Compression reconstruction error of Grouplet-CS transform with adaptive threshold of different compression ratios

In view of the reconstruction error of Ribeira data, this section introduces adaptive projection Bayesian compressed 
sensing algorithm to estimate noise and improve the reliability of information recovery.

\section{B. Application of hyperspectral data compression and} reconstruction

In this section, the application research compares the effects of adaptive threshold and Grouplet transform with fixed hard threshold (the threshold is set to 0 ) on hyperspectral image compression and reconstruction. Mainly used for comparative study: hard threshold Grouplet-FBCS algorithm, hard threshold Grouplet-AFBCS algorithm, adaptive threshold Grouplet-FBCS algorithm and adaptive threshold Grouplet-AFBCS algorithm.

The advantages of the adaptive Grouplet-AFBCS algorithm can be seen from the error results of image information reconstruction in each band. The effectiveness of the algorithm is verified by comparing the reconstruction error of hyperspectral $3 \mathrm{D}$ data with the average peak signal-to-noise ratio, and the reliability of the result is proved by the change trend of structural similarity ratio. As shown in figures 9 and 10 .

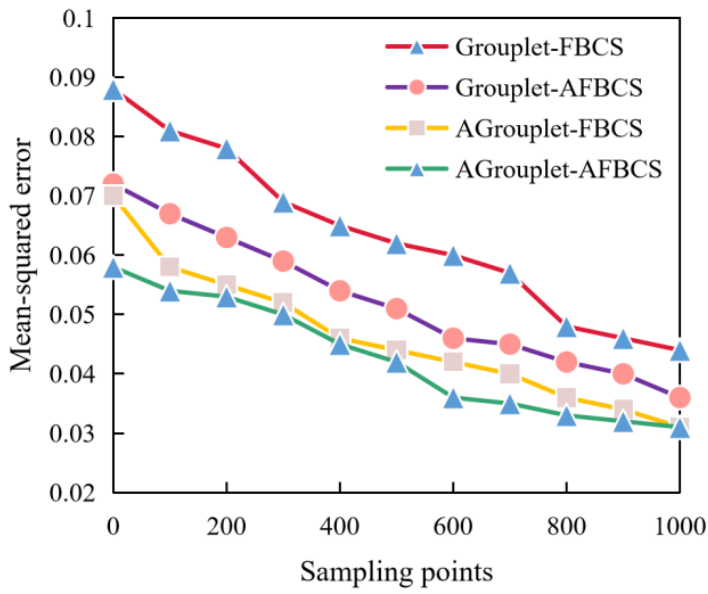

Figure 9. Mean root mean square error line chart of different algorithms and different sampling points

Grouplet transform with adaptive threshold has better performance for sparse model provided for compression reconstruction process when image is sparse. Not only is the sparsity good, but the sparsity coefficient is closer to the original signal.

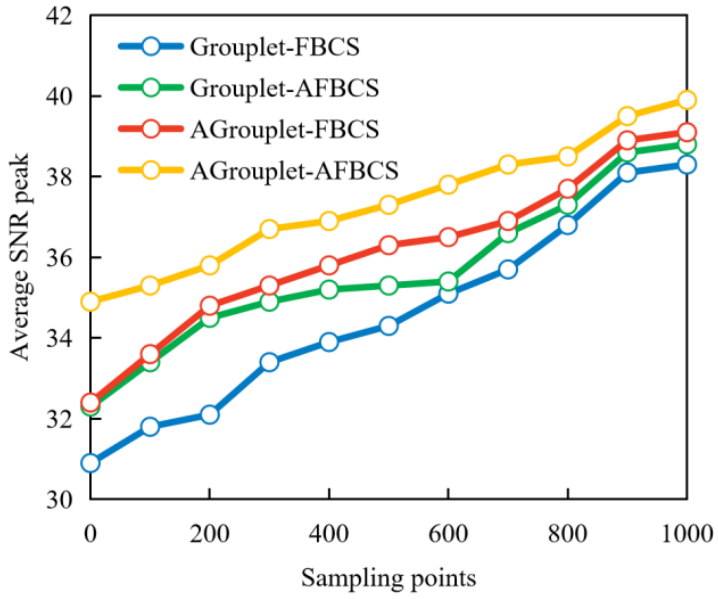

Figure 10. Peak line chart of average signal-to-noise ratio of different algorithms and sampling points

At the same time, it can be seen that the algorithm combined with the adaptive Grouplet transform needs less sampling points. When the sampling points are 600 , the error reduction is no longer obvious, and the peak value of signal-to-noise ratio rises slowly. Therefore, the algorithm not only reduces the error of information recovery, but also shortens the operation time and improves the calculation efficiency. These two pictures also verify that the adaptive FBCS algorithm is superior to FBCS algorithm in sampling points and information reconstruction accuracy.

The reconstruction error and peak signal-to-noise ratio of two different Grouplet bases combined with adaptive FBCS algorithm show a rapid change trend, but when the sampling point is 600 , the change trend gradually slows down. Therefore, the expected expectation is verified, and Figure 11 further clarifies the reliability and effectiveness of the algorithm proposed in this section.

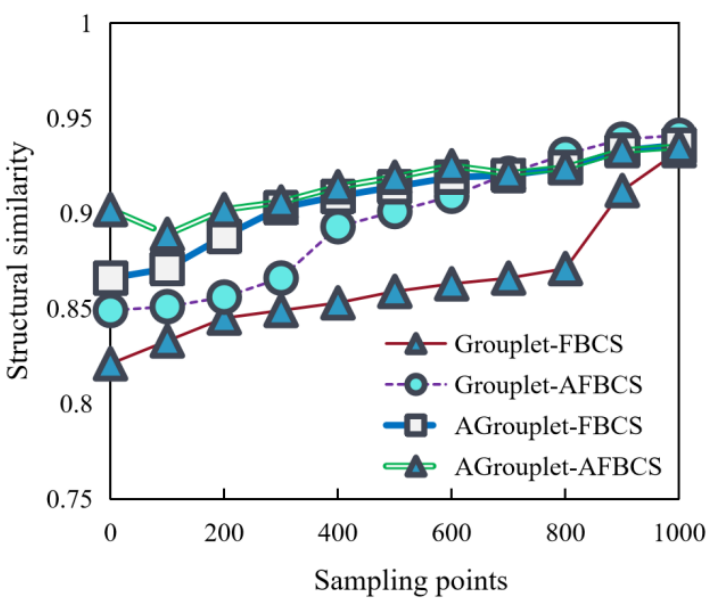

Figure 11. Line chart of structural similarity of different algorithms and different sampling points

Combined with Grouplet basis of different threshold processing methods, hyperspectral images are expressed sparsely, and the image information is reconstructed accurately by Bayesian compressed sensing algorithm and FBCS 
algorithm improved by adaptive projection matrix, so as to obtain high-quality and high-precision reconstruction methods and high signal-to-noise ratio reconstruction results. The feasibility of the proposed algorithm is verified, which shows that the sparse model obtained by Grouplet transform with adaptive threshold has better performance and more reliability.

At the same time, it can be seen that the algorithm combined with the adaptive Grouplet transform needs less sampling points. When the sampling points are 600 , the error reduction is no longer obvious, and the peak value of signal-to-noise ratio rises slowly. Therefore, the algorithm not only reduces the error of information recovery, but also shortens the operation time and improves the calculation efficiency.

This picture also verifies that the adaptive FBCS algorithm is superior to FBCS algorithm in sampling points and information reconstruction accuracy. The reconstruction error and peak signal-to-noise ratio of two different Grouplet bases combined with adaptive FBCS algorithm show a rapid change trend, but when the sampling point is 600 , the change trend gradually slows down. Therefore, the expected expectation is verified.

\section{CONCLUSION}

Hyperspectral imaging technology is a new breakthrough in the development of hyperspectral imaging technology. Studying the information extraction technology in hyperspectral imaging can provide a powerful guarantee for the development and application of interferometric hyperspectral imaging technology. On the basis of studying the evaluation criteria of hyperspectral imaging quality, this paper systematically studies the whole process of data processing and information extraction of interference hyperspectral imaging. A spectral image reconstruction method based on compressed sampling in spatial domain and KL transform in spectral domain is proposed from the perspective of application method, which can effectively reduce the cost of data sampling. There are many bands in hyperspectral images, and the spatial structure of two-dimensional images corresponding to each band is different. Therefore, the adaptive threshold Grouplet transform is combined with adaptive projection Bayesian compressive sensing algorithm, and it is applied to the compression reconstruction of hyperspectral images. The experimental results show that the reconstruction accuracy of this algorithm is better than other algorithms proposed in this paper.

Due to the limited ability and time, my research involves many links such as preprocessing, data inversion, display, transmission and compression, etc., and the research on each link is only superficial, and there is no in-depth and systematic research. For each part of the research, there is a lot of work left behind, which needs to be analyzed and studied in further research.

\section{ACKNOWLEDGMENT}

This research work was supported by a self Funded Project of Langfang Science and Technology Research and Development Plan (the second batch) in 2019, "Design and improvement of directional sound source", No: 2019011045 and Zhao zhen is the Corresponding author.

\section{REFERENCES}

[1] Wang Z, Mi H, Ling W, et al. Semi-NMF-Based Reconstruction for Hyperspectral Compressed Sensing. IEEE Journal of Selected Topics in Applied Earth Observations and Remote Sensing, 2020, PP(99):1-1.

[2] Cui H, Cheng Z, Li P, et al. Prediction of Sweet Corn Seed Germination Based on Hyperspectral Image Technology and Multivariate Data Regression[J]. Sensors, 2020, 20(17):4744.

[3] Junying S U, Yingkui L I, Qingwu H U. A New Spectral-spatial Jointed Hyperspectral Image Classification Approach based on Fractal Dimension Analysis. Fractals, 2019, 27(05):6116-6137.

[4] Guo W, Li X, Xie T. Method and system for nondestructive detection of freshness in Penaeus vannamei based on hyperspectral technology. Aquaculture, 2021, 538(1-2):736512.

[5] Xinghan L, Wenxiu Z, Wenxuan C, et al. Electromagnetic propagation logging while drilling data acquisition method based on undersampling technology. Journal of Geophysics and Engineering, 2021(5):5.

[6] Aghamiri N A, Huth F, Huber A J, et al. Hyperspectral time-domain terahertz nano-imaging. Optics Express, 2019, 27(17):24231.

[7] Ang L M, Seng K P. Meta-scalable Discriminate Analytics for Big Hyperspectral Data and Applications. Expert Systems with Applications, 2021, 176(5):114777.

[8] JM Melián, A Jiménez, M Díaz, et al. Real-Time Hyperspectral Data Transmission for UAV-Based Acquisition Platforms. Remote Sensing, 2021, 13(5):850.

[9] Liu W, Zeng S, Wu G, et al. Rice Seed Purity Identification Technology Using Hyperspectral Image with LASSO Logistic Regression Model. Sensors, 2021, 21(13):4384.

[10] Fu H, Sun G, Ren J, et al. Fusion of PCA and Segmented-PCA Domain Multiscale 2-D-SSA for Effective Spectral-Spatial Feature Extraction and Data Classification in Hyperspectral Imagery[J]. IEEE Transactions on Geoscience and Remote Sensing, 2020, PP(99):1-14.

[11] Mei S, Jiang R, Li X, et al. Spatial and Spectral Joint Super-Resolution Using Convolutional Neural Network. IEEE Transactions on Geoscience and Remote Sensing, 2020, PP(99):1-14.

[12] Wang H, Wang $X$, Chen $C$, et al. Hyperspectral Image Classification Based On Domain Adaptation Broad Learning. IEEE Journal of Selected Topics in Applied Earth Observations and Remote Sensing, 2020, 13(99):3006-3018.

[13] Nardecchia A, Vitale R, Duponchel L. Fusing spectral and spatial information with 2-D stationary wavelet transform (SWT 2-D) for a deeper exploration of spectroscopic images. Talanta, 2020, 224(10):121835.

[14] Zhong S, Zhang Y. An Iterative Training Sample Updating Approach for Domain Adaptation in Hyperspectral Image 
Classification. IEEE Geoscience and Remote Sensing Letters, 2020, PP(99):1-5.

[15] Xie W, Lei J, Cui Y, et al. Hyperspectral Pansharpening With Deep Priors. IEEE Transactions on Neural Networks and Learning Systems, 2019, PP(99):1-15.

[16] Liu W, Lee J. A 3-D Atrous Convolution Neural Network for Hyperspectral Image Denoising. IEEE Transactions on Geoscience and Remote Sensing, 2019:5701-5715.

[17] Signoroni A, Conte M, Plutino A, et al. Spatial-Spectral Evidence of Glare Influence on Hyperspectral Acquisitions. Sensors, 2020, 20(16):4374.

[18] $\mathrm{Hu}$ Y, Li X, Gu Y, et al. Hyperspectral Image Recovery Using Nonconvex Sparsity and Low-Rank Regularizations. IEEE Transactions on Geoscience and Remote Sensing, 2019, PP(99):1-14.

[19] Shi C, Zeng H, Guo Y, et al. Surface Roughness Detection of Roof Insulator Based on Hyperspectral Technology. IEEE Access, 2020, PP(99):1-1.

[20] Uzkent B, Rangnekar A, Hoffman M J. Tracking in Aerial Hyperspectral Videos Using Deep Kernelized Correlation Filters. IEEE Transactions on Geoscience and Remote Sensing, 2019, 57(1):449-461.

[21] Yu W, Zhang M, Shen Y. Spatial Revising Variational Autoencoder-Based Feature Extraction Method for Hyperspectral Images. IEEE Transactions on Geoscience and Remote Sensing, 2020, PP(99):1-14.

[22] Li S, Hu L, Sun C, et al. A Reversible Data Hiding Algorithm Based on Prediction Error With Large Amounts of Data Hiding in Spatial Domain. IEEE Access, 2020, 8:214732-214741.

[23] Fm A, Jma B. Unsupervised exploration of hyperspectral and multispectral images - ScienceDirect. Data Handling in Science and Technology, 2020, 32:93-114.

[24] Tian W, Rui G, Liu G, et al. Efficient Acquisition Method for Marine Monitoring Data Based on Compressed Sensing. IEEE Access, 2019, 7:1-1.

[25] Azizipour M J, Mohamed-Pour K. Compressed downlink channel acquisition based on burst support information in large-scale MIMO systems. Electronics Letters, 2019, 55(8):489-491.

[26] Li C, Qu X, Yang Y, et al. Composite Clustering Sampling Strategy for Multiscale Spectral-Spatial Classification of Hyperspectral Images. Journal of Sensors, 2020, 2020(5):1-17.

\section{Creative Commons Attribution License 4.0 (Attribution 4.0 International, CC BY 4.0) \\ This article is published under the terms of the Creative Commons Attribution License 4.0 \\ https://creativecommons.org/licenses/by/4.0/deed.en_US}

